\title{
Pandemic Influenza and Acute Care Centers: Taking Care of Sick Patients in a Nonhospital Setting
}

Sandro K. Cinti, William Wilkerson, Jennifer G. Holmes, Jean Shlafer, Christopher Kim,

Curtis D. Collins, Kenneth Bandy, Frank Krupansky, Marie Lozon, Stuart A. Bradin, Carrie Wright, Janet Goldberg, Deborah Wagner, Phillip Rodgers, Jenny Atas, and Bruce Cadwallender

The ongoing spread of $\mathrm{H} 5 \mathrm{~N} 1$ avian influenza in Southeast Asia has raised concern about a worldwide influenza pandemic and has made clear the need to plan in advance for such an event. The federal government has stressed the importance of planning and, in particular, has asked hospitals and public health agencies to develop plans to care for patients outside of traditional healthcare settings. These alternative or acute care centers (ACCs) would be opened when hospitals, emergency departments (EDs), and clinics are overwhelmed by an influenza pandemic. The University of Michigan Hospital System (UMHS), a large tertiary care center in southeast Michigan, has been developing a model for offsite care of patients during an influenza pandemic. This article summarizes our planning efforts and the lessons learned from 2 functional exercises over the past 3 years.

$\mathbf{R}^{\mathrm{R}}$ ECENT DISASTERS, including Hurricanes Rita and Katrina, have highlighted the reality that healthcare workers may be asked to evaluate and treat patients in a healthcare setting that is functioning well beyond capacity. ${ }^{1}$ In addition, the ongoing $\mathrm{H} 5 \mathrm{~N} 1$ avian influenza outbreak in Southeast Asia has raised concern about a worldwide influenza pandemic and the need to plan in advance for such an event. ${ }^{2}$ The federal government has stressed the importance of planning and has asked hospitals and public health agencies to develop plans to care for patients outside of traditional healthcare settings. ${ }^{3}$ These alternative care sites would be opened if hospitals, emergency departments (EDs), and clinics become overwhelmed by an influenza pandemic. ${ }^{4}$

Sandro Cinti, MD, is Associate Professor, Infectious Diseases, University of Michigan Hospitals/VA Ann Arbor Health Systems; William Wilkerson, MD, is Associate Professor, Department of Emergency Medicine, University of Michigan; Jennifer G. Holmes, RN, MHSA, is in the Emergency Department, University of Michigan Hospital System; Jean Shlafer, MHSA, is Director of Ancillary Nursing and Admissions, Bed Coordination Center, Nursing Administration, University of Michigan Health System; Christopher Kim, MD, is Assistant Professor, Internal Medicine; University of Michigan; Curtis D. Collins, PharmD, MS, is a Clinical Pharmacist, Department of Pharmacy Services, University of Michigan Health System; Kenneth P. Bandy, BS, RRT, is Director, Respiratory Care Service, University of Michigan Hospitals and Health Centers; Frank Krupansky, MSA, is Director, Materiel Services, University of Michigan Health System; Marie Lozon, MD, is in the Emergency Department, University of Michigan Hospital System; Stuart A. Bradin, DO, is Assistant Professor of Pediatrics and Emergency Medicine, University of Michigan Health System, Department of Emergency Medicine, Division of Pediatric Emergency Medicine; Carrie M. Wright, MS, is an Emergency Management Specialist, Safety Management Services; Janet Goldberg, MSA, RN, is Director of Cancer and Palliative Care Nursing, Nursing Services, University of Michigan Health System; Deborah Wagner, PharmD, is Associate Professor, Department of Pharmacy, University of Michigan Health System; Phillip Rodgers, MD, is in the Department of Family Medicine, University of Michigan Hospital System; and Bruce A. Cadwallender, MS, is Director of Safety and Emergency Management, Safety Management Services, University of Michigan Hospitals and Health Centers; all are in Ann Arbor, Michigan. Jenny Atas, MD, is Region 2 South Medical Director and Associate Professor of Emergency Medicine, Wayne State University, Detroit, Michigan. 
The University of Michigan Health System (UMHS) is a 913-bed tertiary care hospital system with approximately 10,000 employees. Pandemic influenza preparedness has been an ongoing mission at our institution, and improving surge capacity has become a top priority for our Infectious Hazards Group. Like most U.S. hospitals, the UMHS Emergency Department (ED) is always full, and the inpatient census runs near capacity at all times., ${ }^{5,6}$ An influenza pandemic like the one that occurred in 1918 would quickly overwhelm UMHS with sick and concerned patients. Rapid action will be required to free up capacity in our hospital system, including: discharging stable patients, canceling elective surgeries and procedures, canceling nonurgent outpatient visits, and reassigning staff to respond to the surge of influenza patients. In addition, once the hospital has reached capacity, UMHS staff might be required to care for patients in off-site wards or acute care centers (ACCs).

The Office of Public Health Preparedness (OPHP), a division of the Michigan Department of Community Health (MDCH), is responsible for Michigan's response to a public health emergency. After September 11, OPHP partitioned the state into 8 Medical Bio-Defense Networks (MBDN). Each MBDN is headed by a planning board composed of the Medical Control Authorities (MCAs) in that region and is responsible for planning for and coordinating a response to public health disasters. The Region 2
South (R2S) MBDN is the largest network and is composed of 38 hospitals that serve approximately 2.8 million people in southeast Michigan. An advisory group led by a medical director is the operational arm of the R2S MBDN and is composed of representatives from hospitals, public health agencies, emergency management groups, police, and mental health services.

In 2003, OPHP asked all 8 MBDNs in Michigan to develop a Modular Emergency Medical System (MEMS) in order to "facilitate augmentation of local heathcare response efforts" during disasters. ${ }^{8}$ MEMS is based on a military model of treating injured or ill soldiers in the field, but it has been modified to address issues specific to public health disasters. ${ }^{4}$ Specifically, MEMS has been designed to provide mass care for a specific problem (eg, infection) and to provide that care in nonhospital settings, thereby relieving hospitals from being inundated with sick patients. The components of MEMS include (see Figure 1):

- A regional Medical Coordination Center (MCC) that serves as the central point of coordination, supporting local Emergency Operations Centers (EOCs) by managing healthcare resource allocation in a defined geographical area;

- Neighborhood Emergency Help Centers (NEHCs) that serve as the entry points into the medical system for

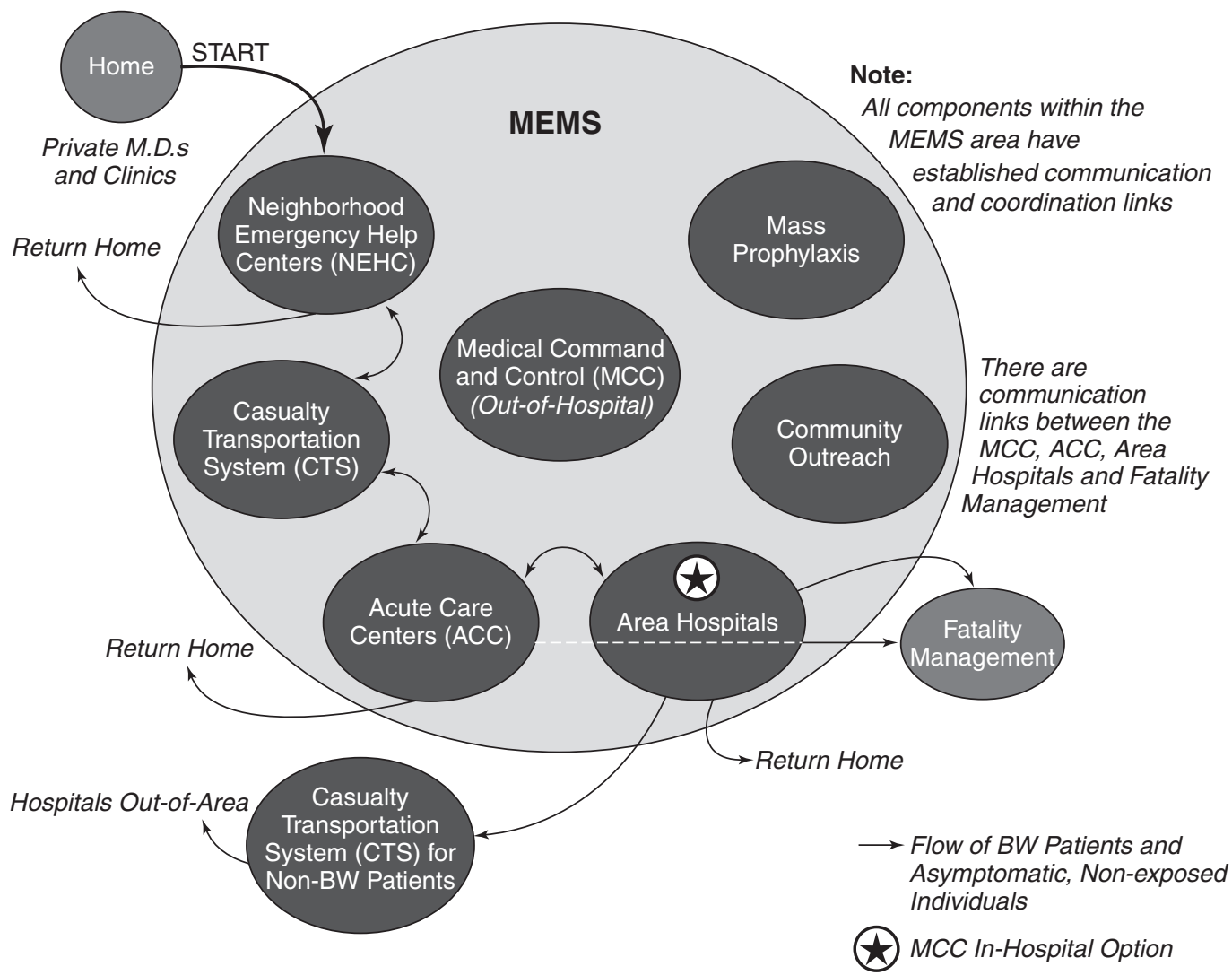

Figure 1. Modular Emergency Medical System (MEMS) Model 
triage of casualties, provision of limited first aid and mass prophylaxis, and information dissemination;

- Acute/Alternative Care Centers (ACCs) that serve as inpatient units during a disaster or pandemic, treating patients who require more extensive medical care such as hydration, oxygen, or intravenous antibiotic therapy; and

- Casualty Transport Systems (CTS), which are designed to move patients within the components of MEMS.

MDCH and the R2S MBDN leadership have asked (and partially funded) each large hospital system within the region to develop plans to operate, staff, and partially supply an ACC that can house up to 250 patients. UMHS has been developing such a plan and has, over the past 3 years, in conjunction with $\mathrm{MDCH}$ and Region 2 South, completed 2 functional exercises to test our capabilities. The following is a summary of the creation and function of an ACC at UMHS and the after-action reports from the 2 functional exercises.

\section{Creation and Function of the ACC}

Alternative care centers are generally defined as "locations, preexisting or created, that serve to expand the capacity of a hospital or community to accommodate or care for patients or to protect the general population from infected individuals during mass casualty incidents." The ACC has had various descriptions in the literature of its location, situation, and functionality. Lam and colleagues tried to clarify the concept of ACCs in various contexts, and they reviewed the literature for the different models. They came up with 7 models that provide some or all of the following services: (1) an overflow hospital providing the full range of care; (2) patient isolation and an alternative to home care for infectious patients; (3) expanded ambulatory care; (4) care for recovering, noninfectious patients; (5) limited supportive care for noncritical patients; (6) primary triage and rapid patient screening; and (7) quarantine. ${ }^{9}$

UMHS decided on an ACC that is most consistent with model 5 but that would also provide palliative care. In consultation with the Region 2 South MBDN and the University of Michigan campus leadership, UMHS selected the Central Campus Recreation Building (CCRB) as its ACC facility. This selection was based on Region 2 South criteria adapted from a multiagency federal document entitled "Acute Care Center, Modular Emergency Medical System: Concept of Operations for the Acute Care Center (ACC)." Criteria included the size of the facility, access for ambulances, proximity to UMHS, electrical and water supply, heating/air conditioning, lighting, and ability to secure the facility. The CCRB is a large, 2-story building with adequate space (145,000 square feet) to accommodate up to 250 patients as well as staff and equipment. The facility has easy access, it is located less than $1 / 2$ mile from UMHS, and its perimeter is easy to secure.

The ACC would be activated once UMHS neared 120\% capacity and would function like an inpatient ward. It would contain 4 pods with 50-60 beds or cots in each, for a total of 200-250 patients, and would provide a limited range of care to pandemic influenza patients who are not critically ill. Each pod would be staffed by teams including physicians, nurses, medical techs, and a pharmacist (see Staffing below). ACC functions included administering IV fluids, IV/oral antibiotics, and antivirals for pandemic influenza victims. Oxygen and breathing treatments were to be provided, but patients with increasing oxygen requirements would be sent to the hospital. The facility also would function as a step-down unit from the hospital, and a portion of the ACC would be dedicated to palliative care of terminally ill patients. It was expected that the average stay would be 3 days, and that over 12 weeks this facility would be able to care for 5,600-7,000 people (given adequate staffing and supplies).

\section{The Functional Exercises}

UMHS and Region 2 South carried out 2 functional exercises in the CCRB in June 2006 (Exercise 1) and May 2007 (Exercise 2). The exercises were limited to the upper floor of the CCRB, which is essentially 4 contiguous basketball courts (Figure 2). Patients (actors) were admitted to one of four 50-bed pods: adult, pediatric, oxygen, or palliative care. During the first exercise, 28 patients were admitted, and 18 were admitted during the second. Fifty cots were assembled in Exercise 1, and 100 were assembled in Exercise 2 to assess spatial limitations. Patients were admitted from Neighborhood Emergency Help Centers, UMHS outpatient clinics, UMHS inpatient units, and the UMHS emergency department. These pre-triaged patients were transported by local ambulances. Also, several patients presented directly to the ACC and had to have a medical screening exam, which was performed by the ACC staff.

Valuable lessons were learned during the 2 functional exercises. The following discussion addresses specific components of the ACC that were tested during the exercises, including location, admitting/patient flow, communication, staffing, supplies and equipment, security, and palliative care. Each component is broken down into the major lessons learned (after-action reports) and changes made after Exercises 1 and 2.

\section{Location}

Both exercises confirmed that the CCRB will make a suitable ACC. The building fulfills most of the ACC criteria outlined above. Specifically, it can accommodate 200-250 


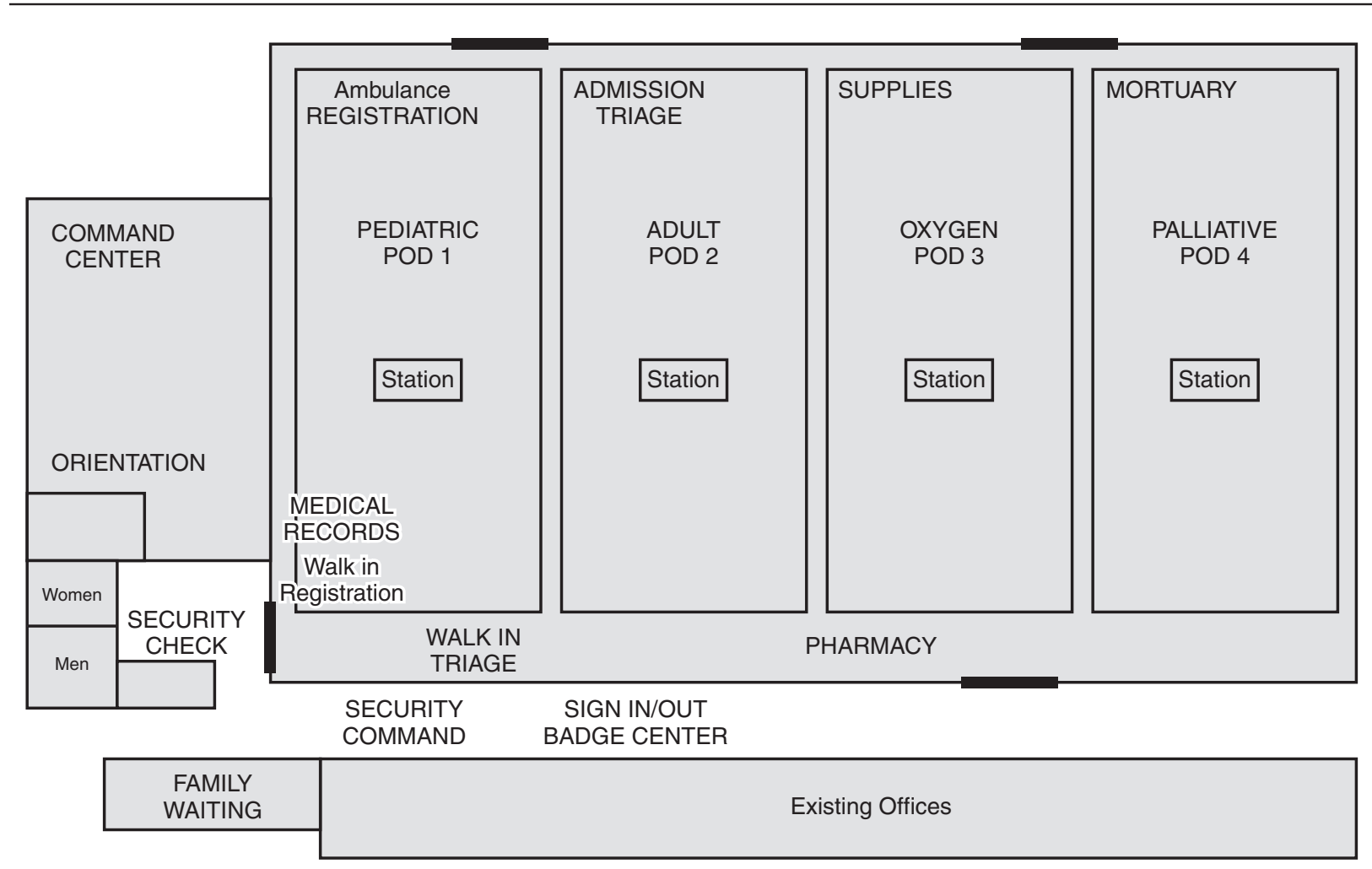

Figure 2. ACC Layout

patients and staff, it is near UMH, it has an adequate water and electrical supply, and it is easy to secure and to control patient flow. In our after-action discussions for both exercises, the difficulty with moving patients from floor to floor, the lack of enough bathrooms, and the lack of air conditioning were identified as problems. These are not insurmountable, however, and we resolved to keep all patients on one floor and to pursue agreements on obtaining portable toilets and cooling systems.

\section{Admitting and Patient Flow}

\section{Exercise 1}

During our first exercise in 2006, the ACC resembled an emergency department more than an inpatient unit. Instead of being directly admitted to a bed, as would occur on a medical ward, patients were first required to go through nursing triage and medical screening exams. The added triage slowed down the movement of patients within the ACC and created a bottleneck in the waiting area. Paper charts were created for patients deemed appropriate for the ACC, and they were admitted to 1 of the 4 pods (adult/family, pediatric, oxygen, or palliative care). Patients who were medically unstable were transferred to the hospital, and those who did not fit ACC criteria, including the "worried well," were sent home or were referred elsewhere. Because the ACC was considered an extension of the UMHS inpatient system, bed managers created an extra 250 "beds" that could be tracked with the 913 UMHS beds. The ACC medical director was responsible for accepting admissions from UMHS or other R2S hospitals.

Lessons Learned from Exercise 1. The major problem identified in the after-action report regarding the admissions process was that the onsite triage process diverted too many resources away from the inpatient function of the ACC. We had patient back-ups in the waiting area, and nurses and physicians could not focus on processing admitted patients. Also, by allowing self-referral to the ACC, we were unable to control the influx of patients.

Changes after Exercise 1. Given these problems in Exercise 1 , we refined our admissions process to mirror an inpatient ward, and we minimized self-referral to the ACC.

\section{Exercise 2}

During Exercise 2, we minimized the number of patients who self-presented to the ACC by creating simulated public announcements and setting up security posts that routed people to preestablished triage centers. For those patients who needed medical screening and nursing triage, we decreased assessment time by implementing a mini-registration process and moving them quickly to a treatment area for full evaluation. We also fine-tuned admissions from outside entities by requiring that all ACC admissions be called into the UMHS Emergency Operations Center (EOC) and approved by an accepting physician. Once the admission was approved, a booking was created to assign the patient to one of the $250 \mathrm{ACC}$ beds and the admission

Biosecurity and Bioterrorism: Biodefense Strategy, Practice, and Science 
was called to the charge nurse at the ACC, who took the report. The lead ACC physician was then called by either the transferring physician or the EOC accepting physician with the specifics of the case.

Lessons Learned from Exercise 2. The after-action report confirmed that this admissions process worked better and that patient flow proceeded more smoothly. There were problems, however, when the patient volume increased. Specifically, the efficient transfer of important clinical information became difficult when multiple patients presented simultaneously. Patients accepted by the EOC physician often presented to the ACC before information had been given to either the charge nurse or the lead ACC physician.

Changes after Exercise 2. We are currently reorganizing the UMHS EOC to include more than one EOC accepting physician in order to accommodate admissions and improve communication with the ACC. Electronic bed monitoring should avoid the problem of overbooking ACC beds from the EOC. We will test this system in the future.

\section{Communications}

\section{Exercise 1}

During the 2006 exercise, most of the effort regarding communication centered on interactions within the ACC. Walkie-talkies were distributed among all staff, and channels were posted on erasable marker boards for certain pathways (eg, channel 23: pharmacy). Communication with outside entities, including the UMHS, occurred via email and cell phones and landlines. However, since most patients self-presented in Exercise 1, we did not have much outside communication other than with the R2S Medical Coordination Center.

Lessons Learned from Exercise 1. In our after-action report, it was clear that, although walkie-talkies worked quite well, coordinating channels was sometimes difficult and staff tended to favor person-to-person communications. We also realized that communication with outside entities was crucial if we were going to change our admissions protocol as outlined above.

Changes after Exercise 1. We resolved to improve intraACC communications by expanding redundant communication modalities. Furthermore, we developed and tested communications between the ACC and outside entities more thoroughly during Exercise 2.

\section{Exercise 2}

During the 2007 exercise, we continued to use walkietalkies with designated channels in our intramural communications. However, we added some redundancy by including several 800-megahertz radios, and all lead staff were given cell phones. Redundancy was extended to outside communications, and we were particularly careful to ensure open lines between the ACC leadership and contacts at the Region 2 South EOC, the UMHS EOC, and the Neighborhood Emergency Help Center, which was staffed by the county public health department. These communications occurred mostly over cell phones, but email and landlines were also essential. In addition, we included a ham radio operator in case other media broke down.

Lessons Learned from Exercise 2. Even with all the redundancy built into our communication system, we still had difficulty both sending and receiving timely information. The after-action report identified communication both inside the ACC and between the ACC and outside entities as a crucial area that required more work. As patient load increased, communications worsened and nerves frayed.

Changes after Exercise 2. UMHS has continued to update its communication capabilities. We have used CDC/HRSA funding to purchase 800-megahertz radios and more cell phones. UMHS is also committed to expanding redundancy in our communication system. We are developing a hospitalwide health alert network that will meld with the R2S and state multimodal health alert network (Michigan-HAN). All of these updated systems will be used in the ACC for intramural and external communication.

\section{Staffing an ACC \\ General}

Staffing during an influenza pandemic poses a daunting challenge. It has been estimated that absenteeism during a pandemic may reach $40-50 \%$ of the work force, as members become ill or incapacitated or need to remain home with family. ${ }^{10,11}$ Staffing an ACC will be especially difficult because very few healthcare workers are accustomed to caring for patients in a nontraditional setting. The traditional model for this type of care has been the military hospital, which relies heavily on expertise in trauma medicine. ${ }^{1}$ Emergency medicine and surgical staff, therefore, have the most experience in this setting. The management of hospitalized influenza patients is quite different and relies on a model of care more similar to standard inpatient medicine.

For both functional exercises, we adhered to a staffing model agreed on at the state and regional levels that conforms to the National Incident Management System's (NIMS) Incident Command Structure (ICS) (Table 1). ${ }^{12}$ Leadership positions included: an ACC group supervisor, a nursing unit leader, a medical operations unit leader, a medical care task force leader, a supply/logistics unit leader, a finance unit leader, and a records/planning unit leader. Job action sheets for these positions have been published. ${ }^{4}$ Both Exercises 1 and 2 focused mainly on physicians, nurses, and pharmacy staff, and these were discussed in our after-action reports. Security is discussed separately (see below). 
Table 1. Minimum Staffing Per 12-Hour Shift in an ACC

\begin{tabular}{|l|c|c|}
\hline Beds & 50-Bed Pod & Total for 250 \\
\hline Physicians & $1-2$ & $5-10$ \\
\hline $\begin{array}{c}\text { Physician assistants or } \\
\text { nurse practitioners }\end{array}$ & 1 & 5 \\
\hline Registered nurses & $4-6$ & $20-30$ \\
\hline Nursing assistants/techs & $4-6$ & $20-30$ \\
\hline Clerks & 2 & 10 \\
\hline Respiratory therapists & 1 & 5 \\
\hline Social work/case manager & 1 & 10 \\
\hline Environmental services & 2 & 10 \\
\hline Pharmacist & 2 & 5 \\
\hline Pharmacy technician & 1 & $15-20$ \\
\hline Security & $3-4$ & 5 \\
\hline Transport officer & 1 & $5-140$ \\
\hline Mental health specialist & 1 & 58 \\
\hline Total & $22-28$ & 5 \\
\hline
\end{tabular}

\section{Exercise 1}

Emergency medicine physicians and nurses made up the bulk of the clinical staff in the ACC during the 2006 exercise. A lead ACC physician and an ACC charge nurse acted as the supervisors. One physician was responsible for each of the 4 pods, and 2 nurses worked in each pod. During Exercise 1, 28 patients with influenza were admitted to the ACC and were distributed among the pods. Two pharmacists were assigned to the ACC, and they distributed medications from a room located across from the patient pods. Medication orders were written on triplicate forms and given to the pharmacists, and medications were subsequently placed in bins labeled with the patient's name. Two clerks created charts, a respiratory therapist was present to manage oxygen, and a security guard monitored the ACC perimeter.

Lessons Learned from Exercise 1. In our after-action report on staffing, the major criticism was that Exercise 1 resembled an emergency department more than an inpatient ward. As a result both physicians and nurses spent too much time performing patient triage and attending to sick walk-in patients. Also, after-action participants felt that the ratio of staff to patients was too high and did not reflect what we would see during a true event when staff shortages would stress the system. Even with adequate staffing, most participants felt that the focus on triage caused the flow of the ACC to break down.

Pharmacy staff felt that having pharmacists located away from the patient pods was counterproductive. While medication security and workspace were adequate, communication among nursing, pharmacy, and medical staff was poor. Pharmacy participants felt that the use of triplicate strip orders was efficient and allowed rapid training of pharmacy staff unfamiliar with the UMHS computer system.

Changes after Exercise 1. In preparing for Exercise 2, our group decided to structure the staffing to be more consistent with an inpatient hospital ward. We felt the staffing ratios reflected in Table 1 should be adhered to as strictly as possible in order to simulate a more realistic scenario. We moved pharmacy personnel into the patient pods so that medications could be distributed more efficiently from repositories pre-filled with formulary medications. Finally, we decided to expand the ability to give oxygen in the ACC and thus asked for more involvement from Respiratory Therapy.

\section{Exercise 2}

During the May 2007 exercise, 18 patients were admitted to the ACC. In addition to an ACC, the public health agency was also testing a Neighborhood Emergency Help Center, which did some triage before sending patients to the ACC. During this exercise, only 1 or 2 patients were permitted to self-present. All other patients were admitted from outside entities (eg, the neighborhood center) and approved by the UMHS EOC. Every effort was made to simulate an inpatient ward, including calling reports to the charge nurses and lead ACC physician. Staffing changes were instituted as described above. Personnel involved in triage in Exercise 1 were reassigned to the pods in Exercise 2. Pharmacy staff were now present in the pods and had more contact with clinicians. An oxygen pod supplied with liquefied oxygen necessitated more involvement by respiratory therapy staff.

Lessons Learned from Exercise 2. In our after-action report, most participants felt that, by minimizing triage, Exercise 2 ran much more smoothly than Exercise 1. Pharmacy personnel were pleased with the new dispensing structure, and order processing went quite well. The clinician-to-patient ratio was still not tested optimally, however, as only 2 physicians and 3 nurses were allowed to "play" while others observed without commenting or helping. Unfortunately, as in Exercise 1, clinical participation in Exercise 2 involved ED personnel almost exclusively. Participants realized that ED staff would be unavailable during a true pandemic influenza outbreak because they would be needed in EDs and urgent care centers. Although social work staff did participate in observing the exercise, our after-action report acknowledged the need to expand mental health and palliative care services in the ACC. Very little exercise time was

Biosecurity and Bioterrorism: Biodefense Strategy, Practice, and Science 
spent on fatality management (see Palliative Care section below).

Changes after Exercise 2. It was very clear that a more organized ACC staffing model was needed. Regarding physicians especially, it was clear that we needed a model that did not rely exclusively on emergency department staff. We are currently developing an ACC staffing model that relies heavily on hospitalists as the lead physicians, and, toward that end, UMHS has designated a hospitalist physician as the ACC medical director. Hospitalists, who have clinical and procedural expertise in the management of acutely ill hospitalized patients (both adult and pediatric) and who have a systems perspective in delivering efficient quality care, are uniquely qualified to develop and operate an ACC. ${ }^{13}$ Additionally, these clinicians are highly skilled at moving patients from inhospital to outpatient care. During an influenza outbreak, an efficient transition of patients from the inpatient to outpatient setting will be essential to limit overflow.

Hospitalists will be supported in the ACC by other medical providers, particularly resident physicians. As almost half of these physicians are rotating on consult services at any particular time, we plan on reassigning these individuals during a pandemic outbreak. Medicine, pediatric, and surgical residents who have completed their intern year are accustomed to caring for acutely ill patients. They are generally proficient at many procedures (eg, central lines) and most are trained in advanced cardiac life support (ACLS). These physicians also are accustomed to managing unstable patients with fluids, oxygen, and occasionally, vasopressors and mechanical ventilation. We intend that our final ACC physician staffing plan will resemble an inpatient team with a faculty-level physician supervising several residents.

We also have designated an ACC nursing lead who is working out a plan to reassign nurses to ACC duty. It is expected that the usual patient-to-nurse ratio will change from the standard $4: 1$ or $6: 1$ in the hospital to between $8: 1$ and 12:1 in an ACC during an influenza pandemic (Table 1). The nursing staff felt that this was achievable provided clearly defined influenza treatment protocols were available. Some routine nursing services, such as vital sign checks every 4 hours, may need to be adapted or delegated to nursing assistants in order to free up registered nurses to provide IV fluid and medication management and ongoing assessment of patient response to treatment. UMHS is also including retired nurses in a volunteer registry.

We also plan to expand the role of home care nurses in a pandemic. This group can help ACC patients make the transition back home. It is our intention, through phone triage banks, to keep as many people home as possible. During the 1918 pandemic, most ill people recovered at home with the help of family, friends, and home visiting healthcare workers. ${ }^{14}$ We intend to use our home care nursing services in a similar fashion by building on recently created pandemic home care models. ${ }^{15}$
It is anticipated that a shift in staff experience will occur, as was reported in a February 2007 Agency for Healthcare Research and Quality (AHRQ) publication. ${ }^{16}$ As the pool of experienced clinicians is depleted, less experienced staff may need to be recruited to staff the ACC. Medical and nursing students in their mid-third and fourth years of training have considerable experience in caring for acutely ill inpatients. In a recent survey, $70 \%$ of medical students believed that healthcare students have a moral, ethical, and professional obligation to volunteer during a pandemic. ${ }^{17}$ During the 1918 influenza pandemic, the graduation of some medical students in the U.S. was expedited in order to increase the number of medical personnel available to care for patients. ${ }^{14}$ More recently, however, during the SARS outbreak in Canada, medical and nursing students were removed from the wards, and their education and research activities were curtailed. ${ }^{18}$

Our ACC staffing plan matches novice clinicians like nursing and medical students with experienced staff in order to ensure continuity of high-level care. We have involved both medical and nursing students in our pandemic influenza planning discussions, and we have met with the leadership of the UM medical and nursing schools to discuss how students might help in a pandemic. Student leaders in the UM medical school have expanded emergency medicine groups to include students interested in biological disasters, and there is an active effort to develop a volunteer pool to help during disasters.

As a result of the after-action report, we are also expanding our staffing plan to include mental health and palliative care experts (see Palliative Care below). Furthermore, the need to provide oxygen in the ACC makes it necessary to expand the respiratory therapist role (see Supply Issues below).

\section{Supplies and Equipment in the ACC}

\section{Exercise 1}

During the 2006 exercise, much of the equipment used was provided by Region 2 South in the form of an ACC trailer. The trailer contained enough supplies and equipment for a 50-bed pod, including cots, personal protective equipment, vascular access supplies, administrative equipment, bandaging supplies, and medications including antibiotics. A patient floor supply cart was also brought to the ACC from UMHS. Ten or more cots were set up in each pod to test space availability and ease of movement. Packaged equipment was not unwrapped, and medications were not opened, but their use was noted as patients were admitted. During Exercise 1, oxygen administration was indicated by cardboard signs next to the patients' beds, and no oxygen system was tested.

Lessons Learned from Exercise 1. In our after-action report, participants felt that there was enough space in the ACC for 
up to 250 cots. There was concern, however, about the ability of the R2S MBDN's ability to provide equipment and supplies during a pandemic. UMHS follows a "just-intime" inventory system based on historical usage levels, so continuing to support our hospitals plus an ACC during a pandemic influenza event presented a number of challenges. In addition, today's healthcare supply chain runs lean, with manufacturers and distributors maintaining limited inventories. Regarding the use of oxygen, our group felt that we needed to provide oxygen for most of our patients, given that many would have pneumonia.

Changes after Exercise 1. UMHS decided that we must be prepared to supply our own ACC, and, thus, we resolved to stockpile up to 250 cots and other equipment. A full stockpile has not yet been developed, but purchases have begun, and key supplies and equipment such as masks, gloves, bottled water, and cots will be held at an off-site facility. We chose not to stockpile all necessary supplies and equipment but rather to negotiate a fee for our distributor to hold these supplies for us-essentially, an insurance policy. We have developed a medication formulary (Table 2; available at http://www.liebertpub.com) that includes antibiotics, antivirals, pain medications, antipyrrhetics, antidiarrheals, and antiemetics. We will hold extra stocks of these ACC medications at UMHS for pandemic influenza. Finally, we resolved to test a liquid oxygen delivery system in Exercise 2.

\section{Exercise 2}

Once again, the R2S ACC equipment and supply trailer was used for this exercise. In addition, UMHS provided 50 of its own cots for the functional exercise. The bed setup was similar to Exercise 1. As before, packaged equipment and medications were not opened but were used in virtual fashion. During this exercise, however, a great deal of effort was dedicated to the oxygen delivery system.

Cryogenic (liquefied) oxygen was selected as the primary source gas because of its ability to provide significant amounts of gaseous oxygen on demand for a significant amount of time. The system includes an 850-gallon, trailermounted cryogenic oxygen system. This system also includes a 5,000 or 10,000 standard cubic feet per hour (scfh) mounted vaporizer and a 6-valve manifold system and can be refilled without interrupting service. The capacity of this system would also provide the capability of providing oxygen service to 100 beds within the ACC at variable flow rates for several days before it needed to be refilled. Portable compressed oxygen cylinders (E size) would be used as an emergency back-up and for the transfer of any patients to the hospital.

Based on the conversion rates of oxygen from liquid to gas, we estimate that an 850-gallon portable cryogenic oxygen system will provide 100 beds within our ACC with continuous oxygen up to a maximum of $6 \mathrm{lpm}$ for 3 days before the system needs to be refilled. This system can be set up within several hours of notification and can be maintained with minimal support.

Lessons Learned from Exercise 2. In our after-action report, participants felt confident that we could provide adequate oxygen support to 100 or more sick patients. Participants acknowledged that UMHS must continue to develop its own stockpile of equipment and supplies and not rely on the $\mathrm{R} 2 \mathrm{~S}$ repository. It was acknowledged that some medications are held in the Strategic National Stockpile (SNS) and that we might have access to those supplies several days to weeks into an influenza pandemic.

Changes after Exercise 2. The UMHS ACC group has assumed much of the responsibility for supplies and equipment. This group was established after Exercise 2 to continue to work on all aspects of ACC preparedness. An ACC equipment list is in draft form, and the ACC medication formulary (see Supplemental Table 2 at www.liebert online.com/doi/pdfplus/10.1089/bsp.2008.0030) is being circulated for comment. Stockpiling space is being created at UMHS for more cots and ACC equipment. Also, a final draft of the oxygen delivery system is being completed, and agreements for its rapid deployment during a pandemic are being procured with a supply company. Although a smaller liquid oxygen system was used during Exercise 2, we are in the process of purchasing a larger system at a cost of between $\$ 50,000$ and $\$ 55,000$.

\section{Security}

\section{Exercise 1}

During the June 2006 exercise, security was mainly used to control the influx of self-presenting patients. A specific scenario involving an unruly patient was played out in order to test the security response. We did very little in the first exercise to test perimeter control.

Lessons Learned from Exercise 1. Although the after-action report acknowledged the success of security measures to control the patient waiting area, we had not tested perimeter control very well.

Changes after Exercise 1. We decided to minimize selfpresenting patients in Exercise 2. This allowed security to focus on perimeter control for the second exercise.

\section{Exercise 2}

During this exercise, security focused on securing the perimeter and preventing unfettered access to the ACC. One security person was assigned to inside duties, and 3 patrolled the perimeter. We did not simulate disorderly conduct, but we did ask security personnel to critically assess our ability to secure the premises.

Lessons Learned from Exercise 2. Our after-action report confirmed that the CCRB is an ideal location from a security standpoint. There is only 1 vehicle access point, and pedestrian traffic can easily be controlled at 3 different en- 
try points. This will likely allow us to use fewer security staff than the 20 per ACC shift we initially calculated.

Changes after Exercise 2. The security plan is being rewritten with more emphasis on perimeter control and rerouting of patients and traffic to other triage sites.

\section{Palliative Care and Fatality Management in the ACC}

Exercises 1 and 2

Although we did designate a palliative care pod as well as a morgue area, we did not actively test this aspect of the ACC. One patient scenario in each exercise did include a death; however, other than transferring those patients to the morgue, very little discussion occurred. Neither the medical examiner nor a pathologist was involved in either exercise.

Lessons Learned. In our after-action report, participants acknowledged our lack of attention to palliative care and fatality management. Although we had discussed using refrigerated trucks to store the deceased, this was not practically tested, and clergy personnel were involved only as observers in both exercises.

Changes after Exercises 1 and 2. Since the 2 exercises, we have begun to focus more attention on both palliative care and fatality management. A fatality management plan has been drafted and includes provisions for tracking deaths in the ACC and storing the deceased in refrigerated trucks. We have involved clergy in discussions about pandemic influenza and included clergy members on the UMHS pandemic influenza group. Most significantly, we have expanded our palliative care model with the help of an expert in that field.

In our after-action report, 3 important issues arose with our ACC palliative care model. First, having a treatment area staffed exclusively to provide palliative care would decrease our flexibility to respond to a variable demand for all beds. The number of dying patients in the ACC would likely fluctuate, perhaps significantly, challenging us to either leave empty beds in the palliative care pod (limiting access at peak demand) or to move dying patients to other areas (difficult if staff and resources are not in place to provide palliative care in all areas).

Second, while it seemed possible to assign clearly dying patients immediately to the palliative care areas at triage, many others would progress there after being admitted in one of the other pods. These patients would then need to be transferred to the palliative care area "to die," a move which could well compound both physical and emotional suffering for patients and families.

Finally, patients who require palliative care are likely to be more seriously ill, and they should perhaps more appropriately be sent to the hospital and not the ACC. As a result, palliative care staff and resources would be needed at UMHS as well to manage the significant physical, emo- tional, and family needs of these patients through the end of their lives.

To address these issues, we have begun developing "mass palliative care protocols," which will be used in UMHS and the ACC to meet patient and family needs throughout both facilities. We have also decided not to designate a geographic area for palliative care in the ACC, but rather to discuss how best to provide as much comfort and dignity as possible to dying patients in the existing treatment areas. Solutions may include specific training for site leads in the use of the palliative care protocols, preferentially triaging palliative care patients to UMHS (and private or semi-private rooms) when possible, and using UMHS palliative care providers as consultants during ACC operation. Finally, we are changing the ACC formulary to include more palliation medications, including pain medications and anti-anxiety medications.

\section{CONCLUSION}

If a 1918-type influenza pandemic were to occur, our already stressed healthcare system would become overwhelmed with sick patients. In addition to developing plans to open up hospital beds, the healthcare system must be prepared to care for patients in nontraditional settings. We have outlined how we established and tested an acute care center at the University of Michigan. After 3 years of planning and exercising, we have learned a great deal about how UMHS might manage a pandemic. It is very clear that developing an ACC is critical, and UMHS has, therefore, convened an ACC group headed by the ACC medical director. This group, which meets every 2-3 months, includes leads in nursing, pharmacy, medicine, security, palliative care, materiel services, and hospital administration. Many challenges remain, but we feel that by continuing to prepare for an inevitable pandemic, UMHS will also be ready for other future healthcare emergencies.

\section{ACKNOWLEDGMENTS}

We would like to acknowledge the hard work done by members of the University of Michigan Pandemic Influenza Committee. Without the contributions of each member, this manuscript would not have been possible.

\section{REFERENCES}

1. Surge Hospitals: Providing Safe Care in Emergencies. Oakbrook Terrace, IL: Joint Commission on Accreditation of Healthcare Organizations; 2006. http://www.jointcommission.org/ PublicPolicy/surge_hospitals.htm. Accessed October 21, 2008. 
2. U.S. Department of Health and Human Services. HHS Pandemic Influenza Plan. Washington, DC: U.S. Department of Health and Human Services; 2005. http://www.hhs.gov/ pandemicflu/plan/. Accessed October 21, 2008.

3. Homeland Security Presidential Directive 21 [news release]. Washington, DC: The White House; October 18, 2007. http://www.whitehouse.gov/news/releases/2007/10/ 20071018-10.html. Accessed October 21, 2008.

4. Skidmore S, Wall WT, Church JK. Acute Care Center, Modular Emergency Medical System: Concept of Operations for the Acute Care Center (ACC): Biological Weapons Improved Response Program. Lebanon, NH: Northern New England Metropolitan Medical Response System; May 2003.

5. Frumkin K. Emergency medicine: our house has many rooms (and they are all full). Ann Emerg Med 2007;49:246.

6. Steele R, Kiss A. EmDOC (Emergency Department Overcrowding) Internet-based safety net research. L Emerg Med 2008 Jul;35(1):101-107.

7. Sobieraj JA, Reyes J, Dunemn KN, et al. Modeling hospital response to mild and severe influenza pandemic scenarios under normal and expanded capacities. Mil Med 2007;172(5): 486-490.

8. Michigan Pandemic Influenza Community Health Plan. July 2008. http://www.michigan.gov/documents/mdch/ MDCH_Pandemic_Influenza_v_3.1_final_draft_060 107 . 2_198392_7.pdf. Accessed October 21, 2008.

9. Lam C, Waldhorn R, Toner E, Inglesby TV, O’Toole T. The prospect of using alternative medical care facilities in an influenza pandemic. Biosecur Bioterror 2006;4(4):384-390.

10. Ehrenstein BP, Hanses F, Salzberger B. Influenza pandemic and professional duty: family or patients first? A survey of hospital employees. BMC Public Health 2006 Dec 28;6:311.

11. Influenza Pandemic Planning. Business Continuity Planning Guide. New Zealand Ministry of Economic Development. www.med.govt.nz/upload/27552/planning-guide.pdf. Accessed October 21, 2008.

12. Burkle FM Jr, Hsu EB, Loehr M, et al. Definition and functions of health unified command and emergency operations centers for large-scale bioevent disasters within the existing ICS. Disaster Med Public Health Prep 2007;1:135-141.

13. Kisuule F, Minter-Jordan M, Zenilman J, Wright SM. Expanding the roles of hospitalist physicians to include public health. L Hosp Med 2007;2(2):93-101.

14. Starr I. Influenza in 1918: recollections of the epidemic in Philadelphia. 1976. Ann Intern Med 2006;145:138-140.

15. McGonagle M. VNAA helps agencies prepare for the worst. Home Health Nurse 2007;25:487.

16. Phillips SJ, Knebel A. Mass Medical Care with Scarce Resources: A Community Planning Guide. Rockville, MD: AHRQ; 2007. http://www.ahrq.gov/research/mce/mceguide. pdf. Accessed October 21, 2008.

17. Herman B, Rosychuk RJ, Bailey T, Lake R, Yonge O, Marrie TJ. Medical students and pandemic influenza. Emerg Infect Dis 2007;13:1781-1783.

18. Clark J. Fear of SARS thwarts medical education in Toronto. BMJ 2003;326:784.

Manuscript submitted June 6, 2008;

accepted for publication September 2, 2008.

Address reprint requests to: Sandro Cinti, MD Associate Professor Infectious Diseases

University of Michigan Hospitals/VA Ann Arbor Health Systems 2215 Fuller Rd. Ann Arbor, MI 48105

Email: scinti@umich.edu 
CINTI ET AL.

\begin{tabular}{|c|c|c|c|c|c|c|c|c|c|}
\hline 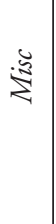 & 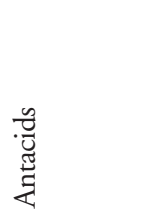 & 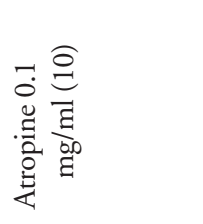 & 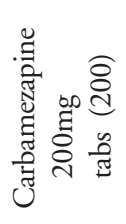 & 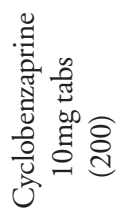 & 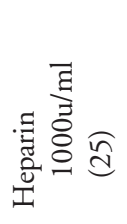 & 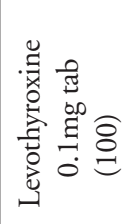 & 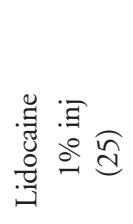 & 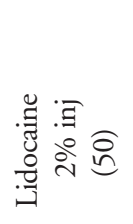 & 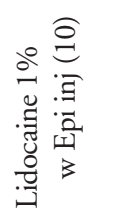 \\
\hline 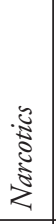 & 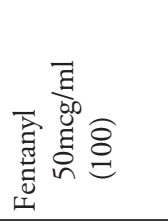 & 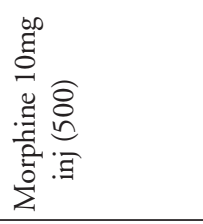 & 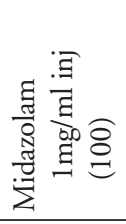 & 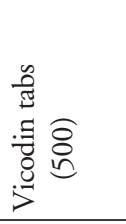 & 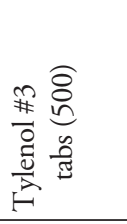 & 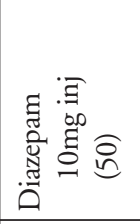 & 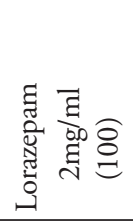 & 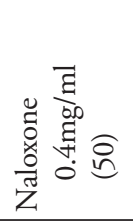 & 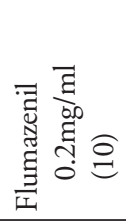 \\
\hline 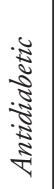 & 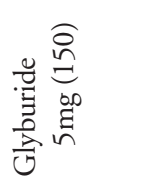 & 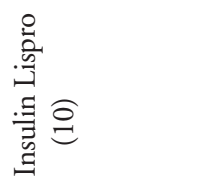 & 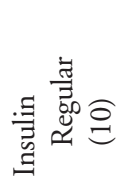 & 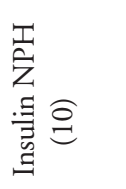 & 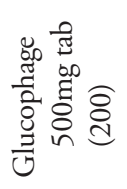 & 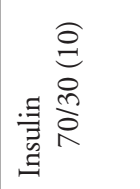 & 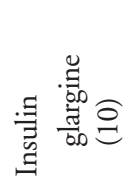 & 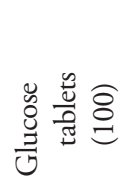 & 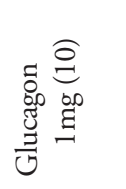 \\
\hline 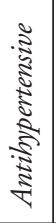 & 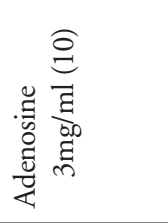 & 志劳总 & & 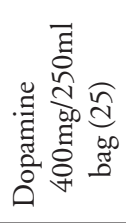 & 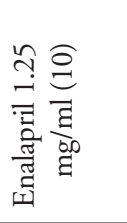 & 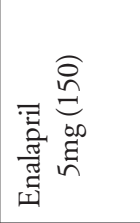 & 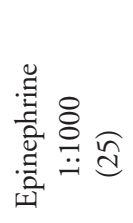 & 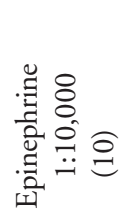 & 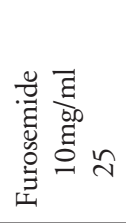 \\
\hline 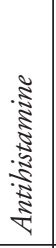 & 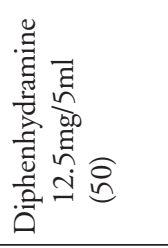 & 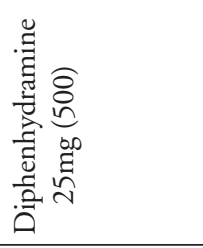 & 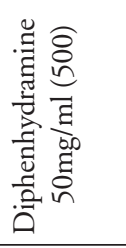 & 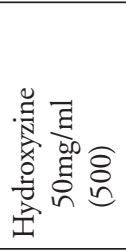 & 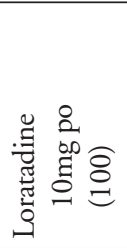 & 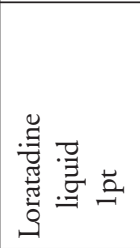 & & & \\
\hline 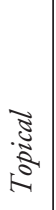 & 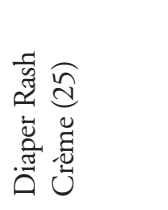 & 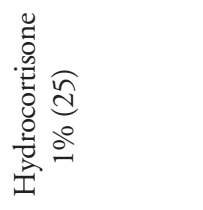 & 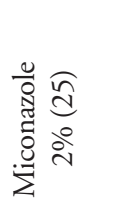 & 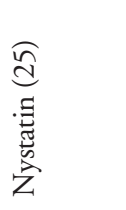 & 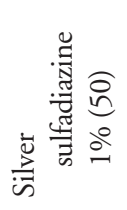 & 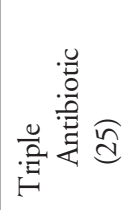 & 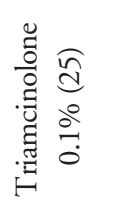 & 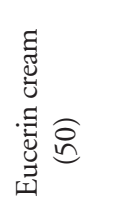 & \\
\hline 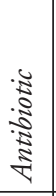 & 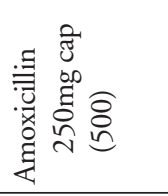 & 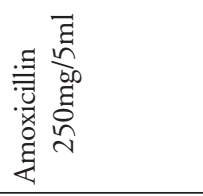 & & 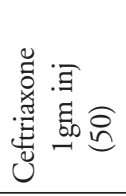 & 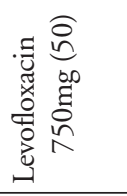 & 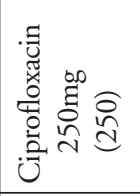 & 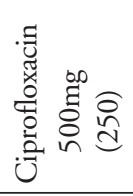 & 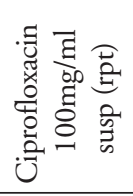 & \\
\hline ৫ & 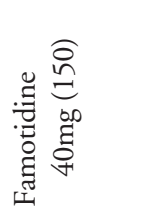 & 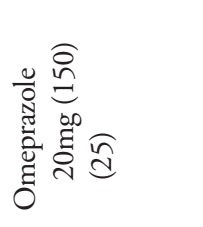 & 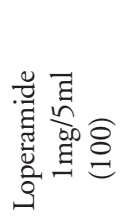 & 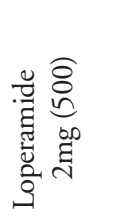 & 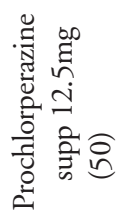 & 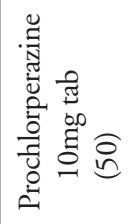 & 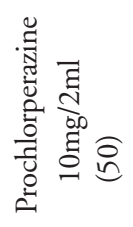 & 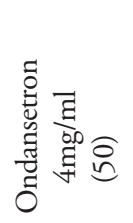 & 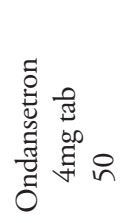 \\
\hline 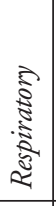 & 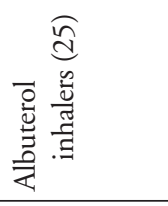 & 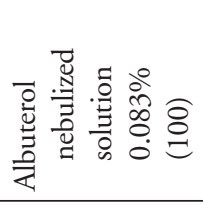 & 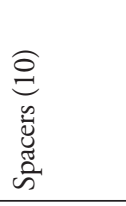 & 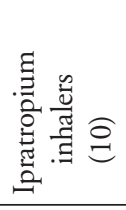 & & & 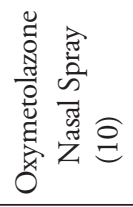 & 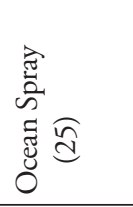 & \\
\hline 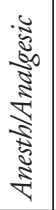 & 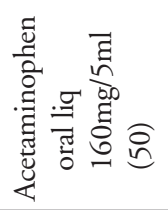 & 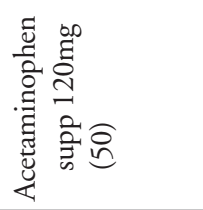 & 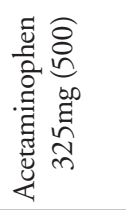 & & 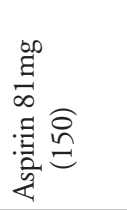 & 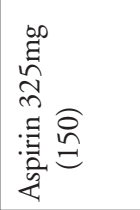 & & 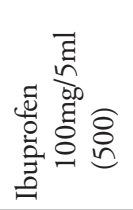 & 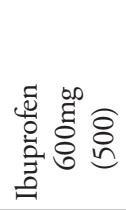 \\
\hline
\end{tabular}


CINTI ET AL.

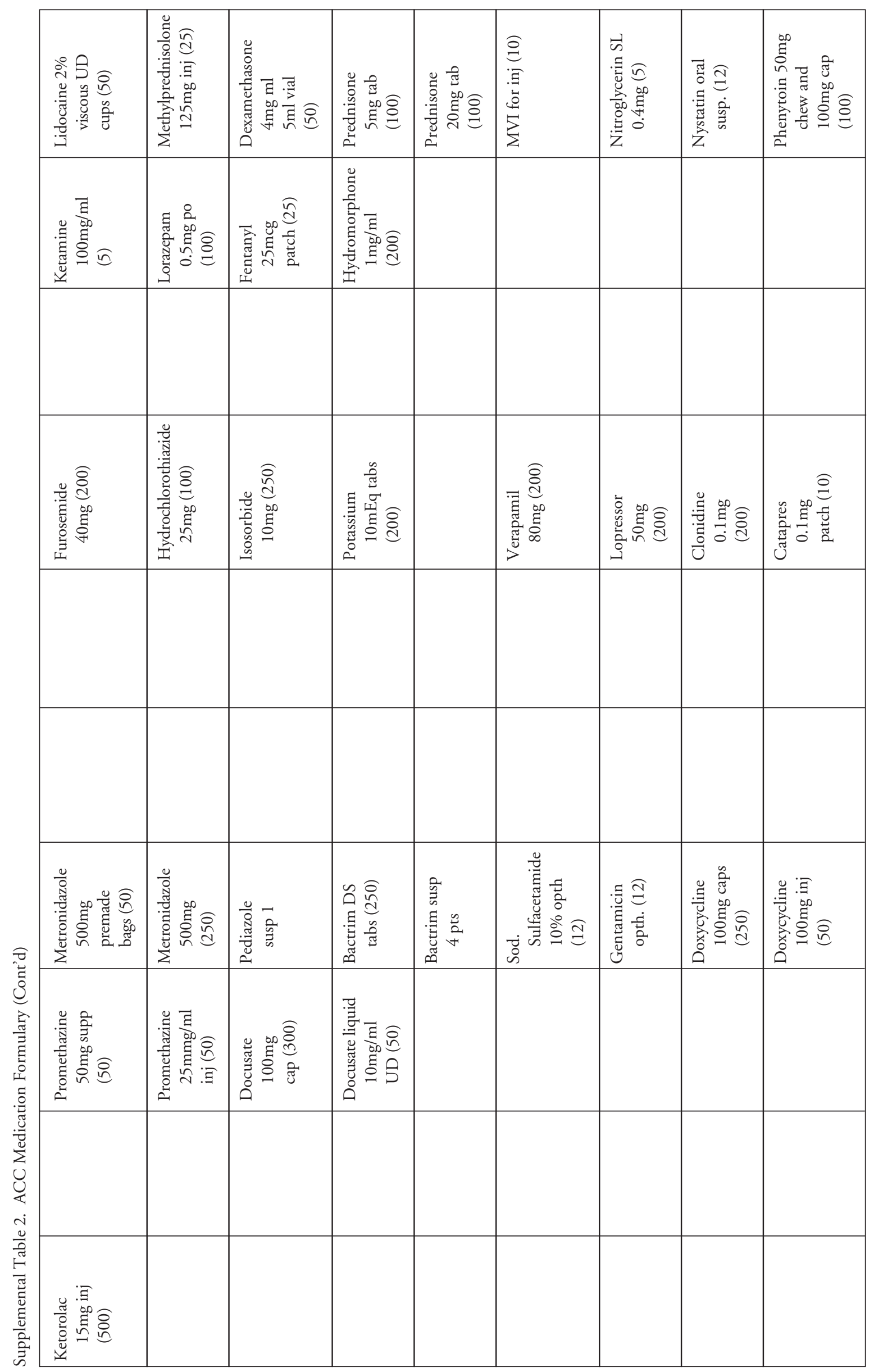


CINTI ET AL.

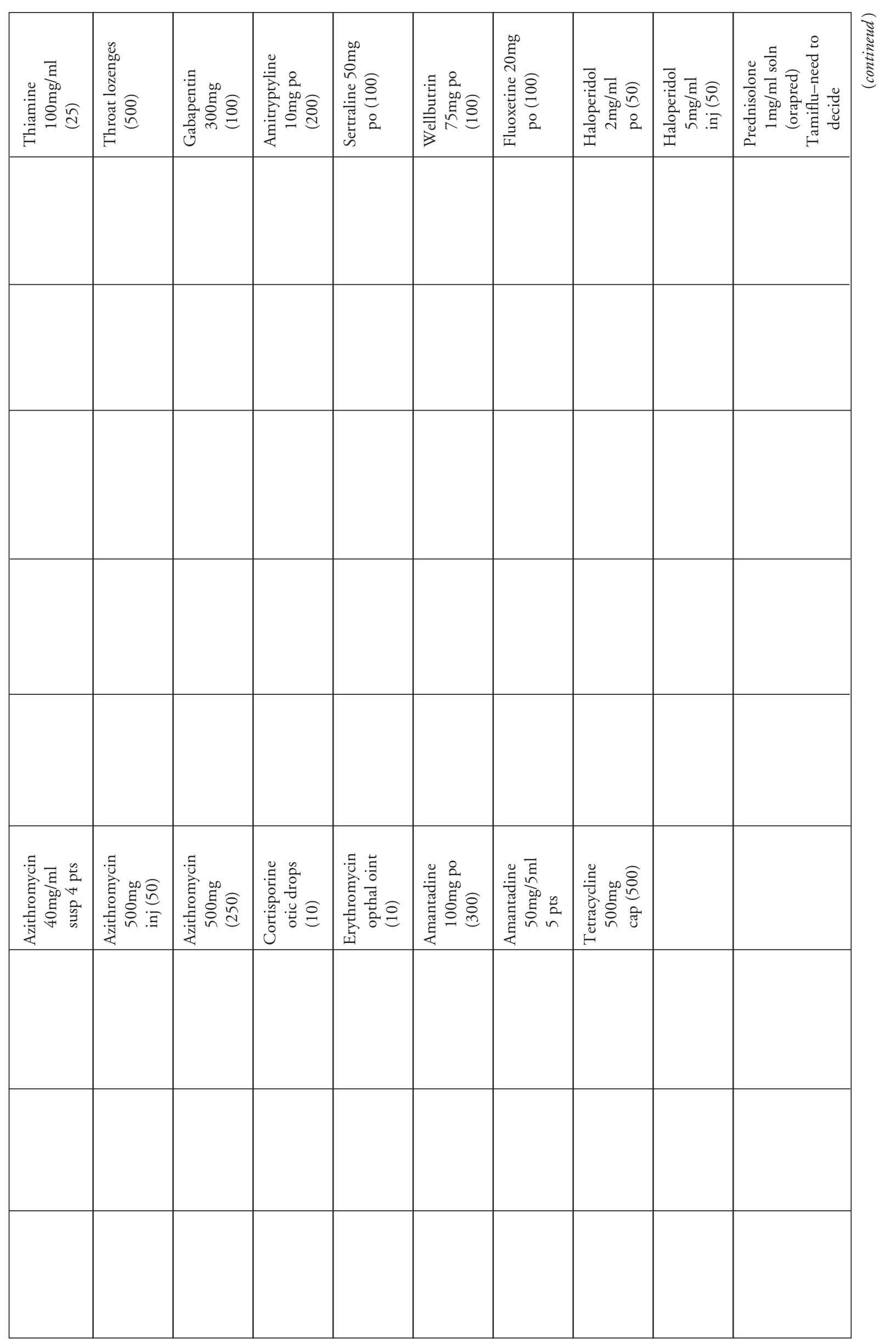


CINTI ET AL.

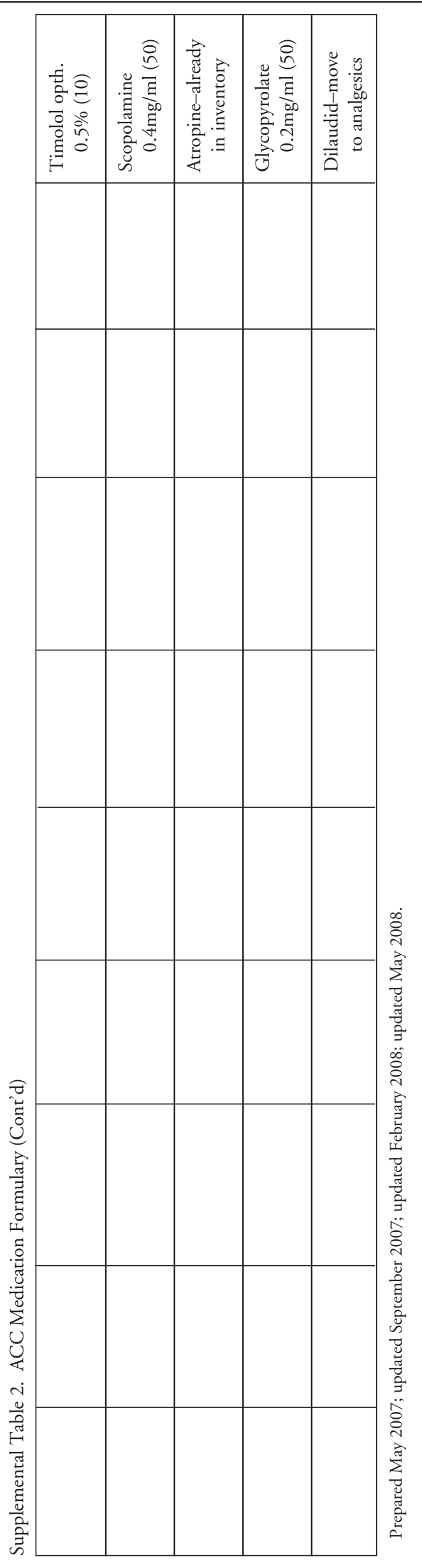


This article has been cited by:

1. Richard Waldhorn . 2008. What Role Can Alternative Care Facilities Play in an Influenza Pandemic? What Role Can Alternative Care Facilities Play in an Influenza Pandemic?. Biosecurity and Bioterrorism 6:4, 357-358. [Citation] [PDF] [PDF Plus] 